\title{
CONTROLE DE VERRUGOSE, MELANOSE E LEPROSE EM LARANJA PERA, COM FUNGICIDAS E ACARICIDA EM MISTURA COM ADUBO FOLIAR
}

\author{
W. BETTIOL'; G.J. de MORAES; C.S. STEULLA JR.; C. NICOLINO; J.A.H. GALVÃO \\ CNPMA/EMBRAPA, C.P. 69, CEP:13820-000 - Jaguariána,SP
}

\begin{abstract}
RSSUMO: Pulverizaçōes com ziram (Rodisan SC), oxicloreto de cobre (Coprantol BR) e 6xido de fembutatina (Torque $500 \mathrm{SC}$ ) em dosagens normais e subdosagens, isoladamente ou em mistura com um adubo foliar originário da fermentação glutâmica do melaço, controlaram de forma efetiva a verrugose, melanose e leprose em laranja pera. A aplicação isolada do resíduo da fermentação glutâmica do melaço, base para diversos adubos foliares, nas dosagens de 0,25\% e 5\% do produto comercial Ajifol, não interferiu na ocorrência de verrugose e melanose. Entretanto, ocasionou um aumento do número de frutos com leprose, do número de lesóes de leprose por fruto e do número de ácaros da leprose (Brevipalpus phoenicis Geipsks) nas folhas e nos frutos.
\end{abstract}

Descritores: controle químico, Elsinoe fawcetti, E. australis, Diaporthe citri, Brevipalpus phoenicis, controle integrado, citros

\section{CONTROL OF CITRUS SCAB, MELANOSE AND LEPROSIS WITH FUNGICIDES AND MITICIDE MIXED TO FOLIAR FERTILIZZER}

\begin{abstract}
Normal dosage and under-dosage sprays of ziram (Rodisan SC); cooper oxychloride (Coprantol BR) and fenbutatin oxide (Torque $500 \mathrm{SC}$ ), isolately or in association with Ajifol, a foliar fertilizer from molasses glutamic fermentation, provided efficient control of scab, melanose and leprosis in 'Pera' orange. Sprays of Ajifol at 0.25 and $5 \%$ had no effect on the rate of occurrence of scab and melanose, but was correlated with increases in the rates of fruits with symptoms of leprosis, number of leprosis spots per fruit, and the number of leprosis mites (Brevipalpus phoenicis Geijskes) on leaves and fruits.
\end{abstract}

Key Words: Chemical control, integrated control, Elsionoe fawcetti, E. australis, Diaporthe citri, Brevipalpus phoenicis, citrus

\section{INTRODUÇÃO}

A verrugose dos citros, causada pelos fungos Elsinoe fawcetti e $E$. australis, se caracteriza por lesões salientes, corticosas e irregulares, usualmente com 1,0 a 3,0 mm de diâmetro, que podem estar agrupadas, cobrindo grande porção do fruto. Frutos novos de todas as variedades, bem como ramos e folhas novas de limões verdadeiros, laranja azeda e tanger murcote são afetados (COORDENADORIA DE ASSISTENCIA TÉCNICA E INTEGRAL - CATI, 1991). A verrugose é também de suma importância por contribuir para o aumento da incidência da leprose dos citros (FEICHTENBERGER et al., 1986).

A melanose, causada pelo fungo Diaporthe citri, se caracteriza por pequenas lesões arredondadas, ligeiramente salientes e de coloração escura nas folhas, ramos e frutos novos. As lesões nos frutos ficam mais visíveis na época da maturação. Podem estar espalhadas, agrupadas ou em cordões, dependendo da distribuição dos esporos do fungo nos frutos pela chuva (CATI, 1991).

O controle dessas doenças é recomendado quando a produção se destina ao mercado de frutas frescas e baseia-se no uso de fungicidas. A época de controle mais indicada é a da florada principal, quando $2 / 3$ das pétalas tenham caído. Uma segunda aplicação é recomendada 4 a 6 semanas após a primeira (CATI, 1991).

A leprose é causada por um vírus, que é transmitido pelo ácaro Brevipalpus phoenicis, que pertence à familia Tenuipalpidae. Os sintomas desta doença se caracterizam pelo aparecimento de

${ }^{1}$ Bolsista do CNPq.

Sci. agric., Piracicaba, 51(3):494-499, set./dez., 1994 
manchas circulares nos frutos, que vão escurecendo com o tempo até se tornarem pardacentas ou pretas, ligeiramente deprimidas e de diâmetro variável, rodeadas por um halo amarelo nas frutas verdes (CATI, 1991). O controle desta doença é normalmente conseguido pelo controle de seu vetor, existindo diversos acaricidas disponíveis no mercado.

Dentre os tratos culturais para citricultura, a adubação foliar vem ganhando espaço. DECHEN \& NEVES (1988) apresentaram diversas considerações sobre a aplicação foliar de nutrientes na cultura. Por ser uma técnica que pode alterar a população de organismos da parte aérea das plantas, há necessidade de se conhecer suas implicações na ocorrência de verrugose, melanose e leprose.

Em relação ao controle da verrugose, diversos autores vêm demonstrando a possibilidade de diminuir a dosagem dos fungicidas recomendados (FEICHTENBERGER et al., 1986; ESKES et al., 1987, FEICHTENBERGER, 1992).

Este trabalho tem por objetivo avaliar o efeito de subdosagens de fungicidas e acaricida, isoladamente e/ou um mistura com o adubo foliar originário da fermentação glutâmica do melaço, sobre a verrugose, a melanose e a leprose na laranja pera.

\section{MATERIAL E MÉTODOS}

Dois ensaios foram conduzidos no município de Limeira/Estado de São Paulo, no período de setembro de 1990 a agosto de 1991 , contando com os mesmos tratamentos e esquema experimental. O ensaio I foi instalado num pomar de Pera Rio sobre limão cravo com 4 anos; o ensaio II em Pera Rio sobre limão cravo com 6 anos.

Os tratamentos foram: 1. testemunha (aplicação de $\mathrm{H}_{2} \mathrm{O}$ ); 2. adubo foliar originário da fermentação glutâmica do melaço-0,25\% (dose recomendada); 3 . controle químico convencional; 4. 3/4 das doses do tratamento 3; 5. 3/4 das doses do tratamento 3 mais o adubo foliar $-0,25 \% ; 6$. $1 / 2$ das doses do tratamento $3 ; 7.1 / 2$ das doses do tratamento 3 mais o adubo foliar- $0,25 \% ; 8$. $1 / 4$ das doses do tratamento $3 ; 9.1 / 4$ das doses do tratamento 3 mais o adubo foliar-0,25\%; 10 . adubo foliar-5\%; 11 . mesmas doses do tratamento 3 mais o adubo foliar- $0,25 \% ; 12.1 / 2$ das doses do tratamento 3 mais o adubo foliar-5\%.

O tratamento convencional (3) constou de 4 pulverizações consecutivas, realizadas em:
25.09 .90 (ensaio I) e 26.09 .90 (ensaio 2) com ziram (Rodisan SC, $300 \mathrm{ml} / 100 \mathrm{l}$ ) + óxido de fembutatina (Torque $500 \mathrm{SC}, 60 \mathrm{ml} / 100 \mathrm{l}$ ); 30.10 .90 (ensaio I) e $\mathbf{3 1 . 1 0 . 9 0 ~ ( e n s a i o ~ 2 ) ~ c o m ~}$ oxicloreto de cobre (Coprantol, $350 \mathrm{~g} / 100 \mathrm{l})+$ óxido de fembutatina (Torque $500 \mathrm{sC}, 60 \mathrm{ml} / 100$ 1); 12.03 .91 (ensaio I) e 13.03.91 (ensaio 2) com óxido de fembutatina (Torque $500 \mathrm{SC}, 60 \mathrm{ml} / 100$ 1 de água); 22.05 .91 (ensaio I) e 23.05.91 (ensaio 2) com óxido de fembutatina (Torque $500 \mathrm{SC}, 60$ $\mathrm{ml} / 100 \mathrm{l}$ ). O Coprantol BR tem $840 \mathrm{~g} / \mathrm{Kg}$ de oxicloreto de cobre; o Torque $500 \mathrm{SC}$ tem $500 \mathrm{~g} / 1$ de óxido de fembutatina e o Rodisan SC tem 500 $\mathrm{g} / 1$ de ziram.

As pulverizações foram realizadas com auxílio de pistola, na vazão de $101 /$ minuto. $O$ tempo de aplicação foi de 20 segundos/planta. $O$ delineamento experimental foi de 5 blocos ao acaso, tendo cada parcela constituída por 4 plantas em linha. A avaliação foi efetuada nas 2 laranjeiras centrais.

Para verrugose e melanose foram avaliados 100 frutos por planta sendo $25 \mathrm{em}$ cada quadrante, entre 9 e 16.07.91, através de uma escala de notas de 0 a 6 , correspondendo cada nota a $0 ; 0,5 ; 2,5 ; 6,0 ; 12,5 ; 25$ e $50 \%$ da área infectada, respectivamente (FEICHTENBERGER et al., 1986).

Em relação à leprose, avaliaram-se os níveis de ácaros vetores e seus predadores em folhas e frutos, e a intensidade dos sintomas da doença nos frutos. A flutuação dos ácaros nas folhas foi avaliada através de coletas quinzenais de 3 folhas da região mediana de cada quadrante das plantas dos tratamentos 1,2 e 3 . As folhas foram acondicionadas em caixa de isopor para transportar ao laboratório onde o número de ácaros foi avaliado. As avaliações nos demais tratamentos foram feitas apenas em 2 ocasiōes: $25.09 .90 \mathrm{e}$ 04.06.91.

A flutuação dos ácaros nos frutos foi estudada analisando-se 4 frutos tomados ao acaso em cada parcela, 2 frutos por planta e 2 visadas em cada fruto.

A avaliação dos sintomas de leprose dos frutos foi realizada entre 09 e 16.07.91, tomandose 50 frutos por parcela e determinando-se o número de lesões por fruto.

\section{RESULTADOS E DISCUSSÃO}

A aplicação do adubo foliar originário da fermentação glutâmica do melaço a $0,25 \%$ e 
$5 \%$, isoladamente, não controlou a verrugose ou a melanose, como se observa na avaliação da porcentagem de área infectada e da porcentagem de frutos com mais de $2,5 \%$ de sua área infectada (TABELAS 1 e 2). A aplicação do adubo foliar àquelas concentrações, quando misturado com os fungicidas nas dosagens recomendadas $e$ nas subdosagens, não interferiu no controle da verrugose e da melanose (TABELAS 1 e 2). Assim, o adubo foliar utilizado não tem ação direta sobre a verrugose e a melanose e não promove a ação mais eficiente dos fungicidas. Com pulverizações, quando $2 / 3$ das pétalas da florada principal tinham caido e 35 dias após, o controle da verrugose com sub-dosagens (até metade da recomendada) de ziram e oxicloreto de cobre foi semelhante ao controle obtido com as dosagens recomendadas. Resultados semelhantes foram obti- dos quando aqueles produtos, nas mesmas subdosagens, foram misturados com o adubo foliar a 0,25 ou $5 \%$ (TABELAS 1 e 2). Esses dados concordam com os obtidos por FEICHTENBERGER (1992) para verrugose em limoeiro siciliano, indicando que esses produtos podem controlar eficientemente as duas doenças quando aplicados a dosagens abaixo das recomendadas $e$ com FEICHTENBERGER et al., (1986) que observaram que há possibilidade de reduzir a dosagem de diversos fungicidas para o controle da verrugose. ESKES et al., (1987) demonstraram a eficiência de diversos fungicidas no controle da verrugose e também verificaram que 2 aplicações de captafol ou ziram, em dose baixa, foram mais eficientes que uma em dose maior na época da florada. Informações sobre a possibilidade de redução de doses são apresentadas por CATI (1991).

TABELA 1. Efeito de Ajifol em combinação com fungicidas na ocorrência de verrugose e melanose em laranja pera avaliado através da porcentagem de área infectada*

\begin{tabular}{lcccc}
\hline & \multicolumn{2}{c}{ Ensaio I } & \multicolumn{2}{c}{ Ensaio II } \\
Tratamentos & verrugose & melanose & verrugose & melanose \\
\hline 1. Testemunha & $3,123 \mathrm{a} *$ & $2,638 \mathrm{a}$ & $0,435 \mathrm{a}$ & $1,426 \mathrm{a}$ \\
2. Ajifol 0,25\% & $3,050 \mathrm{a}$ & $2,449 \mathrm{a}$ & $0,282 \mathrm{~b}$ & $1,300 \mathrm{ab}$ \\
3. Controle Químico Convencionar & $1,111 \mathrm{ef}$ & $1,505 \mathrm{c}$ & $0,042 \mathrm{e}$ & $1,048 \mathrm{~cd}$ \\
4. 3/4 da dosagem do tratamento 3 & $0,968 \mathrm{e}$ & $1,855 \mathrm{~b}$ & $0,118 \mathrm{de}$ & $1,135 \mathrm{bcd}$ \\
5. 3/4 da dosagem tratam. 3 + Ajifol 0,25\% & $1,341 \mathrm{f}$ & $1,939 \mathrm{~b}$ & $0,111 \mathrm{de}$ & $1,157 \mathrm{bcd}$ \\
6. 1/2 da dosagem do tratamento 3 & $1,124 \mathrm{ef}$ & $1,863 \mathrm{~b}$ & $\mathbf{0 , 1 4 9 \mathrm { cd }}$ & $0,976 \mathrm{~d}$ \\
7. $1 / 2$ da dosagem tratam. 3 + Ajifol 0,25\% & $1,371 \mathrm{~d}$ & $1,798 \mathrm{~b}$ & $0,178 \mathrm{~cd}$ & $1,130 \mathrm{bcd}$ \\
8. 1/4 da dosagem do tratamento 3 & $1,806 \mathrm{c}$ & $1,860 \mathrm{~b}$ & $0,160 \mathrm{~cd}$ & $1,227 \mathrm{abc}$ \\
9. 1/4 da dosagem tratam. 3 + Ajifol 0,25\% & $1,739 \mathrm{c}$ & $2,070 \mathrm{~b}$ & $0,287 \mathrm{c}$ & $1,173 \mathrm{bcd}$ \\
10. Ajifol 5\% & $2,653 \mathrm{~b}$ & $2.628 \mathrm{a}$ & $0,100 \mathrm{de}$ & $1,315 \mathrm{ab}$ \\
11. Dosagem do tratamento 3 + Ajifol 0,25\% & $1,395 \mathrm{~d}$ & $1,990 \mathrm{~b}$ & $0,105 \mathrm{de}$ & $1,113 \mathrm{bcd}$ \\
12. 1/2 dosagem tratam. 3 + Ajifol 5\% & $1,200 \mathrm{def}$ & $2,473 \mathrm{a}$ & $0,073 \mathrm{de}$ & $1,151 \mathrm{abcd}$ \\
\hline C.V. & 7,21 & 6,57 & 10,21 & 8,63 \\
\hline
\end{tabular}

"área infectada: Notas variando de 0 a 6 , onde $0=$ sem infecção e de 1 a $6=0,5 ; 2,5 ; 6,0 ; 12,5 ; 25$ e $50 \%$ da área infectada, respectivamente.

* Média de 1000 frutos. Para análise estatística, os dados foram transformados em arc sen $(x+0,5) / 100$. Médias seguidas de mesma letra (na coluna) não diferem entre si (Duncan 5\%).

- Constou de 4 pulverizações: 25.09 .90 (ensaio I) e 26.09 .90 (ensaio II) com ziram (Rodisan SC, 300 $\mathrm{ml} / 100 \mathrm{l}$ ) + 6́xido de fembutatina (Torque $500 \mathrm{SC}, 60 \mathrm{ml} / 100$ 1); 30.10 .90 (ensaio I) e 31.10 .90 (ensaio II) com oxicloreto de cobre (Coprantol, $350 \mathrm{~g} / 100 \mathrm{l}$ ) + óx. de fembutatina (Torque $500 \mathrm{SC}, 60 \mathrm{ml} / 100 \mathrm{l}$ ); 12.03.91 (ensaio I) e 13.03.91 (ensaio II) com óx. de fembutatina (30 ml/100 1); 22.05 .91 (ensaio I) e 23.05 .91 (ensaio II) com $6 x$. de fembutatina $(30 \mathrm{ml} / 100 \mathrm{l})$. 
TABELA 2. Efeito de Ajifol em combinação com fungicidas na ocorrência de verrugose e melanose em laranja pera avaliado através da porcentagem de frutos com área infectada à $2,5 \%$.

\begin{tabular}{lllll}
\hline & \multicolumn{2}{c}{ Ensaio I } & \multicolumn{2}{c}{ Ensaio II } \\
Tratamentos & verrugose & melanose & verrugose & melanose \\
\hline 1. Testemunha & $83,8 \mathrm{a}^{*}$ & $73,2 \mathrm{abc}$ & $20,3 \mathrm{a}$ & $44,3 \mathrm{ab}$ \\
2. Ajifol 0,25\% & $87,0 \mathrm{a}$ & $77,5 \mathrm{ab}$ & $10,0 \mathrm{~b}$ & $43,1 \mathrm{ab}$ \\
3. Controle Químico Convencionar & $32,2 \mathrm{ef}$ & $57,7 \mathrm{de}$ & $1,2 \mathrm{~d}$ & $40,2 \mathrm{bc}$ \\
4. 3/4 da dosagem do tratamento 3 & $29,4 \mathrm{f}$ & $62,5 \mathrm{de}$ & $3,6 \mathrm{c}$ & $40,5 \mathrm{abc}$ \\
5. 3/4 da dosagem tratam. 3 + Ajifol 0,25\% & $40,2 \mathrm{~d}$ & $61,6 \mathrm{de}$ & $2,0 \mathrm{~cd}$ & $37,7 \mathrm{bc}$ \\
6. 1/2 da dosagem do tratamento 3 & $35,4 \mathrm{def}$ & $61,1 \mathrm{de}$ & $2,5 \mathrm{~cd}$ & $35,1 \mathrm{bc}$ \\
7. 1/2 da dosagem tratam. 3 + Ajifol 0,25\% & $44,5 \mathrm{~d}$ & $56,8 \mathrm{e}$ & $4,3 \mathrm{c}$ & $37,9 \mathrm{bc}$ \\
8. 1/4 da dosagem do tratamento 3 & $57,4 \mathrm{c}$ & $60,6 \mathrm{de}$ & $3,5 \mathrm{c}$ & $40,9 \mathrm{abc}$ \\
9. 1/4 da dosagem tratam. 3 + Ajifol 0,25\% & $57,0 \mathrm{c}$ & $68,3 \mathrm{bcd}$ & $5,0 \mathrm{c}$ & $38,8 \mathrm{bc}$ \\
10. Ajipol 5\% & $77,4 \mathrm{~b}$ & $80,0 \mathrm{a}$ & $3,1 \mathrm{~cd}$ & $49,4 \mathrm{a}$ \\
11. Dosagem do tratamento 3 + Ajifol 0,25\% & $42,3 \mathrm{~d}$ & $66,3 \mathrm{cde}$ & $2,3 \mathrm{~cd}$ & $42,2 \mathrm{ab}$ \\
12. 1/2 dosagem tratam. 3 + Ajifol 5\% & $37,7 \mathrm{de}$ & $82,1 \mathrm{a}$ & $2,6 \mathrm{~cd}$ & $31,4 \mathrm{c}$ \\
\hline C.V. & 10,63 & 12,84 & 34,85 & 16,72 \\
\hline
\end{tabular}

- Médias de 1000 frutos. Para análise estatística, os dados foram transformados em arc sen $(x+0,5) / 100$. Médias seguidas de mesma letra (na coluna) não diferem entre si (Duncan 5\%).

- Constou de 4 pulverizações: 25.09 .90 (ensaio I) e 26.09 .90 (ensaio II) com ziram (Rodisan SC, 300 $\mathrm{ml} / 100 \mathrm{l}$ ) + ́xido de fembutatina (Torque $500 \mathrm{SC}, 60 \mathrm{ml} / 100$ l); 30.10 .90 (ensaio I) e 31.10 .90 (ensaio II) com oxicloreto de cobre (Coprantol, $350 \mathrm{~g} / 100 \mathrm{l}$ ) + óx. de fembutatina (Torque $500 \mathrm{SC}, 60 \mathrm{ml} / 100 \mathrm{l}$ ); 12.03.91 (ensaio I) e 13.03.91 (ensaio II) com óx.de fembutatina $(30 \mathrm{ml} / 100$ 1); 22.05 .91 (ensaio I) e 23.05 .91 (ensaio II) com óx. de fembutatina $(30 \mathrm{~m} / 100 \mathrm{l})$.

Os níveis de ácaros no ensaio I foram marcadamente menores que os do ensaio II (Figura 1). Consequentemente, uma incidência menor de sintomas da leprose foi verificada no ensaio I que no ensaio II (TABELA 3). A aplicação do adubo foliar a 0,25 e $5 \%$, isoladamente, aumentou a incidência de leprose. Entretanto, quando misturado com acaricida, o adubo foliar não interferiu no controle (TABELA 3). Verificou-se que com a aplicação do adubo foliar ocorreu um aumento no nível do ácaro da leprose.

Aplicação de óxido de fembutatina, com ou sem adubo foliar, a até $3 / 4$ da dose recomendada, foram os tratamentos que apresentaram o melhor controle da leprose.
Entretanto, verificou-se uma redução da incidência de leprose, de 19,6 na testemunha para 2,6 com $1 / 4$ da dose recomendada do acaricida, com ou sem adubo foliar. Esse resultado indica a necessidade de se estudar mais detalhadamente quais seriam as melhores doses do óxido de fembutatina para essa finalidade. Além de redução de custos, o uso de menores doses resultará em menor risco de contaminação ambiente.

A aplicação de óxido de fembutatina resultou numa considerável redução dos níveis de ácaros predadores das familias Phytoseiidade e Stigmaeidae. Observou-se, entretanto, uma considerável redução dos níveis do ácaro da leprose. 
TABELA 3. Efeito de Ajifol em combinação com fungicidas e acaricidas na incidência da leprose em laranja pera.

\begin{tabular}{|c|c|c|c|c|c|c|}
\hline \multirow[b]{2}{*}{ Tratamentos } & \multicolumn{3}{|c|}{ Ensaio I } & \multicolumn{3}{|c|}{ Ensaio II } \\
\hline & $\begin{array}{l}\text { \% frutos } \\
\text { c/leprose }\end{array}$ & $\begin{array}{l}\text { lesões/ } \\
\text { fruto }\end{array}$ & $\begin{array}{l}\text { lesões/fruto } \\
\text { lesionado }\end{array}$ & $\begin{array}{l}\text { \% frutos } \\
\text { c/leprose }\end{array}$ & $\begin{array}{l}\text { lesões/ } \\
\text { fruto }\end{array}$ & $\begin{array}{c}\text { lesões/fruto } \\
\text { lesionado }\end{array}$ \\
\hline 1. Testemunha & $2,2 b c^{*}$ & $0,198 \mathrm{ab}$ & $4,45 \mathrm{a}$ & $19,6 \mathrm{a}$ & $2,93 \mathrm{a}$ & $13,06 \mathrm{a}$ \\
\hline 2. Ajifol $0,25 \%$ & $4,2 \mathrm{abc}$ & $0,156 \mathrm{~b}$ & $2,73 \mathrm{ab}$ & $28,2 \mathrm{a}$ & $3,25 \mathrm{a}$ & $12,52 \mathrm{a}$ \\
\hline 3. Controle Químico Convencional & $3,4 \mathrm{bc}$ & $0,106 \mathrm{~b}$ & $1,30 \mathrm{ab}$ & $0,2 \mathrm{c}$ & $0,008 b$ & $0,40 \mathrm{~cd}$ \\
\hline 4. $3 / 4$ da dosagem do tratamento 3 & $4,0 \mathrm{abc}$ & $0,120 \mathrm{~b}$ & $1,63 a b$ & $0,4 \mathrm{c}$ & $0,01 \mathrm{~b}$ & $0,25 \mathrm{~cd}$ \\
\hline 5. $3 / 4$ da dosagem tratam. $3+$ Ajifol $0,25 \%$ & $6,0 \mathrm{ab}$ & $0,280 \mathrm{ab}$ & $1,93 \mathrm{ab}$ & $0,2 \mathrm{c}$ & $0,002 \mathrm{~b}$ & $0,10 \mathrm{~d}$ \\
\hline 6. $1 / 2$ da dosagem do tratamento 3 & $5,0 \mathrm{ab}$ & $0,172 \mathrm{ab}$ & $2,15 \mathrm{ab}$ & $3,2 \mathrm{~b}$ & $0,134 \mathrm{~b}$ & $2,20 \mathrm{bcd}$ \\
\hline 7. $1 / 2$ da dosagem tratam. $3+$ Ajifol $0,25 \%$ & $2,0 \mathrm{bc}$ & $0,066 \mathrm{~b}$ & $0,50 \mathrm{~b}$ & $5,2 b$ & $0,396 \mathrm{~b}$ & $4,94 \mathrm{~b}$ \\
\hline 8. $1 / 4$ da dosagem do tratamento 3 & $1,2 \mathrm{c}$ & $0,026 \mathrm{~b}$ & $0,72 b$ & $1,6 \mathrm{c}$ & $0,084 b$ & $3,63 \mathrm{bc}$ \\
\hline 9. $1 / 4$ da dosagem tratam. $3+$ Ajifol $0,25 \%$ & $3,2 a b c$ & $0,112 \mathrm{~b}$ & $1,82 a b$ & $2,6 \mathrm{~b}$ & $0,332 \mathrm{~b}$ & $7,68 \mathrm{~b}$ \\
\hline 10. Ajipol $5 \%$ & $8,6 a$ & $0,454 a$ & 3,05a & $30,4 a$ & $4,732 \mathrm{a}$ & $13,27 \mathrm{a}$ \\
\hline 11. Dosagem do tratamento $3+$ Ajifol $0,25 \%$ & $4,0 \mathrm{abc}$ & $0,122 \mathrm{~b}$ & $1,64 a b$ & $0,0 \mathrm{c}$ & $0 \mathrm{~b}$ & $0,0 \mathrm{~d}$ \\
\hline 12. $1 / 2$ dosagem tratam. $3+$ Ajifol $5 \%$ & $3,4 \mathrm{abc}$ & $0,052 b$ & 3,03ab & 3,4b & $0,456 \mathrm{~b}$ & $7,08 \mathrm{bc}$ \\
\hline C.v. & 60,21 & 18,51 & 59,49 & 62,99 & 42,67 & 52,03 \\
\hline
\end{tabular}

* Para análise estatistica os dados foram transformados em arc sen raiz $(x+0,5) / 100$. As médias seguidas de mesma letra, na coluna, não diferem entre si (Duncan $5 \%$ ).

- Constou de 4 pulverizaçōes: 25.09 .90 (ensaio I) e 26.09 .90 (ensaio II) com ziram (Rodisan SC, $300 \mathrm{ml} / 100 \mathrm{l}$ ) + óxido de fembutatina (Torque $500 \mathrm{sC}, 60 \mathrm{ml} / 100 \mathrm{I}$ ); 30.10 .90 (ensaio I) e 31.10 .90 (ensaio II) com oxicloreto de cobre (Coprantol, $350 \mathrm{~g} / 100 \mathrm{l}$ ) + óx. de fembutatina (Torque $500 \mathrm{SC}, 60 \mathrm{ml} / 100 \mathrm{l}$ ); 12.03 .91 (ensaio I) e 13.03 .91 (ensaio II) com óx.de fembutatina $(30 \mathrm{ml} / 100 \mathrm{l}$ ); 22.05 .91 (ensaio I) e 23.05 .91 (ensaio II) com óx. de fembutatina (30 ml/100 l).

A redução dos níveis de Phytoseiidae e Stigmaeidae nas parcelas tratadas poderiam ser consequência do efeito direto dos produtos sobre esses predadores, ou uma consequência da redução dos níveis populacionais de outros grupos de ácaros dos quais aqueles predadores se alimentam. $O$ adubo foliar não afetou o nível populacional dos predadores mas aumentou o do ácaro da leprose. Os números de ácaros pertencentes a outras espécies (Panonychus citri, Phyllocoptruta oleivora e Tetranychus sp.) foram muito reduzidos para permitir qualquer conclusão.

\section{CONCLUSÕES}

- a aplicação isolada do resíduo da fermentação glutâmica do melaço, base para diversos adubos foliares, nas dosagens de 0,25 e $5 \%$, não interferiu na ocorrência de verrugose e melanose, mas correspondeu a um aumento do número de frutos com leprose;

Sci. agric., Piracicaba, 51(3):494-499, set./dez., 1994
- a aplicação do adubo foliar em mistura com o óxido de fembutatina não interferiu no controle da leprose;

- o controle da verrugose com subdosagens (até metade da recomendada) de ziram e oxicloreto de cobre foi semelhante ao controle obtido com as dosagens recomendadas;

- a aplicação de subdosagens de 6xido de fembutatina foi efetiva no controle da leprose.

\section{REFERÊNCIAS BIBLIOGRÁFICAS}

COORDENADORIA DE ASSISTÊNCIA TÉCNICA INTEGRAL. Recomendaçōes para o controle das principais pragas e doenças em pomares do estado de São Paulo 1990/1991. Campinas, 1994. 55p. (CATI, Boletim Técnico, 165).

DECHEN, A. R.; NEVES, E. S. V. J. Aplicaçāo foliar de nutrientes em citros. Laranja, Cordeirópolis, v.9, n.1, p.65-92, 1988 . 
FEICHTENBERGER, E.; ESKES, A.B.; PRATES, H.S.; SABATINI, V. Resultados de experimentos recentes sobre o controle da verrugose e melanose dos citros. Laranja, Cordeirópolis, v.7, p.173-207, 1986.

ESKES, A.B.; FEICHTENBERGER, E.; PRATES, H.S. Efeito de dose, época e número de aplicaçōes de fungicidas no controle de verrugose de citros. Summa Phytopathologica, Piracicaba, v.13, p.34, 1987.
FEICHTENBERGER, E. Avaliaçāo de dose, época e número de aplicaçōes de fungicidas no controle de verrugose em limoeiro siciliano. Summa Phytopathologica, Piracicaba, v.18, p.28, 1992.

Recebido para publicação em 20.01.94 Aceito para publicação em 10.03.94 